\title{
Proposta de Interface para Um Sistema Inteligente para Gerenciamento de Energia Fotovoltaica no Contexto Brasileiro
}

\author{
Minjae Lins Chung \\ Instituto Federal do Amazonas - IFAM \\ Manaus, AM, Brasil \\ 2020001395@ifam.edu.br \\ Vitor Bremgartner da Frota \\ Instituto Federal do Amazonas - IFAM \\ Manaus, AM, Brasil \\ vitorbref@ifam.edu.br
}

\author{
Priscila Silva Fernandes \\ Instituto Federal do Amazonas - IFAM \\ Manaus, AM, Brasil \\ priscila.fernandes@ifam.edu.br \\ Luis Rivero \\ PPGCC/UFMA \\ São Luis, MA, Brasil \\ luis.rivero@ufma.br
}

\begin{abstract}
Considering the imbalance between economic development and the environment, there is a concern with the preservation of the planet's natural resources and the use of renewable and sustainable energies. In this context, new systems have emerged to assist in the management of photovoltaic energy. This paper presents a proposal for an intelligent interface for a Web system to monitor, report and consult on the production of energy and health of photovoltaic panels. To raise the requirements and propose the system's graphical interface, the following techniques were used: (a) benchmarking, in which the functionalities of the largest competitors in the market were compared; and (b) document analysis, where comments on competing systems on their social networks were analyzed. The prototype presented allows to observe the production of energy in different periods, besides allowing to check the health of a panel, as well as its individual performance.
\end{abstract}

\section{PALAVRAS-CHAVE}

Automação de Sistemas, Engenharia de Software, Sistemas de Informação

\section{INTRODUÇÃO}

O Brasil é um país que possui grande diversidade de características climáticas e geográficas, contudo é notável que a média anual de irradiação global apresenta boa uniformidade, com médias anuais relativamente altas em todo território nacional [1]. Segundo pesquisas realizadas por Fagundes (2012), o estado de Santa Catarina é o local com a menor radiação solar global do país, no valor de 4,25 $\mathrm{kWh} / \mathrm{m} 2$, sendo este, quatro vezes superior ao apresentado para o território da Alemanha, país líder mundial no aproveitamento de energia solar até o ano de 2014, sendo ultrapassado pela China em 2015, ambos os países permanecem líderes mundiais [2, 3]. A utilização de energias renováveis pode reduzir nos custos operacionais e de dependências, dos já limitados combustíveis não renováveis, além de trazer mais comodidade para os usuários desses sistemas e uma opção mais sustentável para o planeta.

Sistemas de produção de energia renovável envolvem sensores que são acoplados aos equipamentos de geração, transmissão e distribuição de energia, além de sistemas web e aplicativos móveis para o usuário gerenciar e acompanhar a utilização e produção dos equipamentos. Esses dispositivos auxiliam no monitoramento e controle dos equipamentos de forma remota e em tempo real. Uma interface sofisticada que atua como ponte entre sensores e usuários finais pode impactar positivamente na user experience e se tornar uma ferramenta diária de interação humano computador. Algumas empresas oferecem esses produtos e serviços, porém os custos de contratação podem ser elevados.

Para auxiliar no gerenciamento da energia fotovoltaica, este artigo apresenta uma proposta de interface para um sistema web inteligente de baixo custo, com finalidade de monitorar, reportar e consultar sobre a produção de energia e saúde de painéis fotovoltaicos. O procedimento metodológico adotado no desenvolvimento dessa pesquisa consiste em seis etapas. As etapas já realizadas foram conduzidas utilizando-se a metodologia ágil Scrum [4] e procedimentos da área de Engenharia de Software.

O restante desse artigo está organizado da seguinte forma: A Seção 2 apresenta o sistema, metodologia utilizada e o desenvolvimento dos trabalhos. A Seção 3 discorre sobre as considerações finais.

\section{O SISTEMA}

O objetivo da pesquisa é desenvolver um sistema web de baixo custo ao usuário para atender os sistemas de geração de energia. A ideia é que a interface seja adaptável a qualquer tipo de painel existente. O sistema poderá, inclusive, ser utilizado por usuários que construíram seu próprio sistema de geração de energia fotovoltaica.

A metodologia utilizada para o desenvolvimento desta pesquisa consiste em cinco etapas: (1) Elicitação de requisitos, (2) Construção do protótipo, (3) Avaliação do protótipo, (4) Desenvolvimento do sistema, (5) Estudo de viabilidade, e (6) Estudo de caso.

$\mathrm{Na}$ etapa de Elicitação de requisitos, foram aplicadas as técnicas de benchmarking e análise de documentação existente. Foram avaliadas soluções já presentes no mercado brasileiro, como a SolarEdge [5] SolarView [6] e FusionSolar [7]. Na aplicação do benchmarking foram verificadas features como: produção de energia atual, produção de energia por período, previsão climática, informativo individual para cada painel, comparativo de gastos economizados, aviso para danos ou sujeira no painel, e adição manual de painéis por rede local. Na análise de documentação existente, foram avaliados comentários nas redes sociais da solução existente, informações do website e reviews de usuários do produto. 
A partir do benchmarking e da análise da documentos, foram levantados os requisitos para o sistema web. Em seguida, os requisitos foram concretizados por meio da plataforma Figma [8], onde o processo de desenvolvimento das soluções foi iniciado. O desenvolvimento do protótipo de alto nível teve como tecnologia empregada a plataforma Figma, auxiliado da metodologia Scrum para gerenciar o processo de desenvolvimento do protótipo. O Figma, que "é um serviço de vetor online para desenvolvimento de interface e prototipagem com a capacidade de organização colaborativa, desenvolvido pela empresa de mesmo nome" [8]. A plataforma Figma é gratuita e fornece todas as ferramentas necessárias para a fase de design de protótipos, simples ou complexos, incluindo ferramentas vetoriais que são capazes de ilustração sofisticadas, bem como recursos de exportação de código Cascading Style Sheets (CSS) e interação para múltiplos usuários dentro de um mesmo projeto. Já a metodologia Scrum é uma estrutura leve que ajuda pessoas, equipes e organizações a gerar valor por meio de soluções adaptativas para problemas complexos [4], e por isso foi empregada na construção deste protótipo.

A prototipação e avaliação do protótipo, terceira etapa da metodologia, foram realizados de forma iterativa. O protótipo passou pela análise de um profissional da área de IHC (Interação HumanoComputador) com mais de oito anos de experiência na indústria de desenvolvimento de sistemas interativos em empresas nacionais e multinacionais, para avaliação e sugestões sobre a interface e funcionalidade do projeto.

O desenvolvimento da interface do protótipo (Figura 1) priorizou o fácil acesso visual para com os detalhes de produção energética, assim como notificações sobre a saúde e integridade de cada painel solar pertencente ao array (Figura 2).

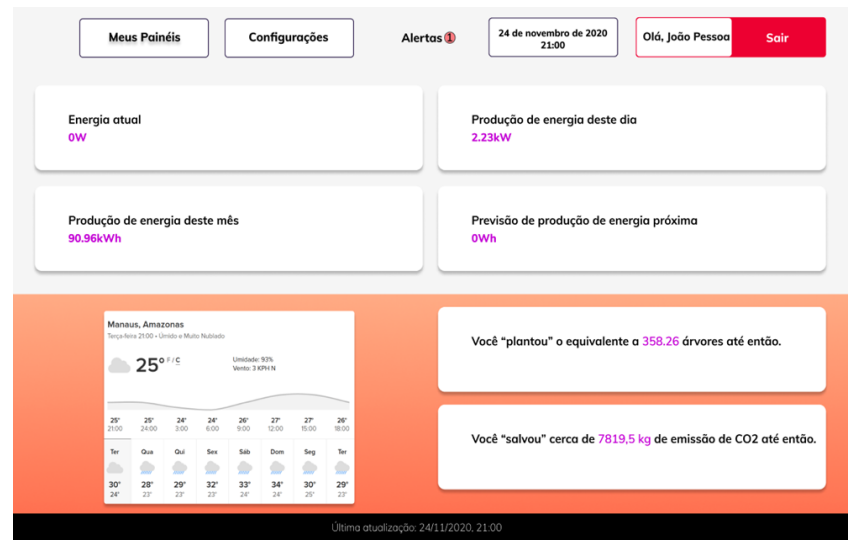

Figura 1: Tela Inicial do Protótipo.

Outras funcionalidades, como previsão climática e alerta para notificações também estão inclusas no protótipo. Estes elementos tiveram seus designs concebidos com base em nove das dez Heurísticas de Nielsen [9], que é um método de avaliação de usabilidade, desenvolvida por Nielsen e Molich, consistindo na inspeção da interface do utilizador no que diz respeito à usabilidade de um software interativo. O método tem se tornado popular, sendo utilizado em vários tipos de sistemas, como sistemas Web, Mobile, Desktop, entre outros [10].

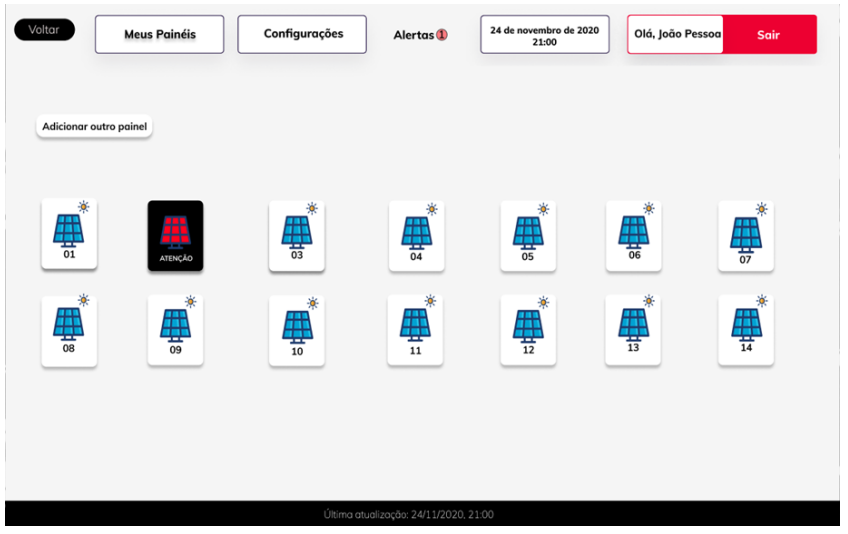

Figura 2: Interface de saúde dos painéis.

As informações disponíveis nas telas foram dispostas conforme a primeira heurística (visibilidade do status do sistema). As cores fortes para informações importantes seguiram a segunda heurística (correspondência entre o sistema e o mundo real). Os menus de: meus painéis, configurações, alertas e sair, sempre estão presentes em todas as telas do sistema e cumprem a terceira heurística (controle e liberdade do usuário) ao dar autonomia para o usuário explorar o sistema sem receios de desconfigurar o mesmo ou se perder dentro de muitas opções. Em todas as telas, os menus ícones e fontes permanecem homogêneos para cumprir com a quarta heurística (consistência e padrões). O menu associado com a opção de sair do sistema é visualmente chamativo, e sempre há a presença do botão de "voltar" nas demais telas, a fim de atender a quinta heurística (prevenção de erros). A presença constante e destacada dos menus principais na tela inicial fornece uma navegação visual para as demais telas do sistema, exercendo assim a sexta heurística (reconhecimento em vez de memorização). A sétima heurística (flexibilidade e eficiência de uso) foi usada ao se pensar na navegação por meio de combinação de teclas para facilitar a experiência de usuários avançados. O design minimalista foi inteiramente pensando na oitava heurística (estética e design minimalista). Por último, a nona heurística (ajude os usuários a reconhecer, diagnosticar e recuperar-se de erros) foi utilizada na inclusão do menu de alerta (Figura 3), localizado no centro de todas as telas, que possibilita visualizar uma notificação que informa: o painel danificado, contato do técnico responsável pela manutenção dos painéis e o estado da saúde do painel em notificação.

\section{CONSIDERAÇÕES FINAIS}

Esse artigo apresentou a proposta de um protótipo de um sistema web de baixo custo para gerenciamento de painéis de geração de energia fotovoltaica. Neste artigo foi descrita condução das três primeiras etapas da metodologia: elicitação de requisitos, prototipação e avaliação do protótipo.

Com a proposta de interface do protótipo finalizada, os próximos passos consistem em desenvolvimento do sistema e realização de dois estudos experimentais.

Os resultados obtidos até o momento foram alcançados com sucesso e praticidade ao aplicar a metodologia Scrum e as Heurísticas 


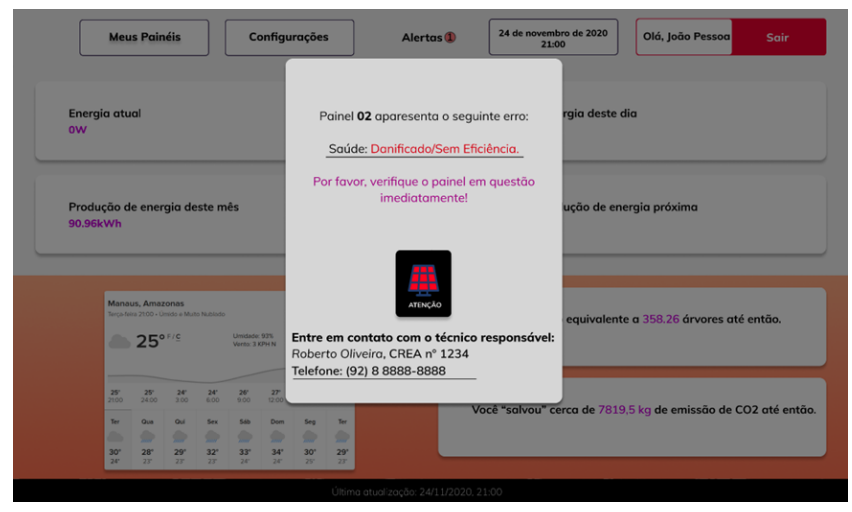

Figura 3: Notificação de alerta

de Nielsen. É esperado que esta solução possa servir como porta de entrada para uma maior utilização da energia solar em residências, visto que implementações como esta, por mais que complexas para iniciantes no mundo da programação, apresentam vantagens econômicas em comparação ao que é ofertado atualmente no mercado. Como trabalhos futuros, pode-se citar o desenvolvimento de um sistema de baixo custo, incluindo os painéis e todo o hardware necessário, para geração de energia solar.

\section{AGRADECIMENTOS}

Este trabalho foi realizado com o apoio do Projeto Aranouá, convênio realizado entre o Instituto Federal do Amazonas (IFAM) e a Samsung Eletrônica da Amazônia (SEDA).

\section{REFERÊNCIAS}

[1] Fagundes Bandeira. Aproveitamento da energia solar no brasil: Aproveitamento e perspectivas. 2012. Disponível em: https://bit.ly/3qWQ0bv. Acesso em: 08 março 2021.

[2] Pedro Daniel da Cunha Kemerich, Carlos Eduardo Balestrin Flores, Willian Fernando de Borba, Rafael Borth da Silveira, Jacson Rodrigues França, and Natalie Levandoski. Paradigmas da energia solar no brasil e no mundo. Revista Eletrônica em Gestão, Educação e Tecnologia Ambiental, 20(1):241-247, 2016.

[3] Orlando Moreira Júnior and Celso Correia de Souza. Aproveitamento fotovoltaico, análise comparativa entre brasil e alemanha. Interações (Campo Grande), 21(2): 379-387, 2020.

[4] Kenneth S Rubin. Essential Scrum: A practical guide to the most popular Agile process. Addison-Wesley, 2012.

[5] SolarEdge. Solaredge: Architects of energy. 2021. Disponível em: https://bit.ly/3bN2rlF. Acesso em: 01 fevereiro 2021

[6] Solarview. Solarview. 2021. Disponível em: https://solarview.com.br. Acesso em: 13 de março 2021.

[7] Fusionsolar. Fusionsolar. 2021. Disponível em: https://www.fusionsolar.eu/. Acesso em: 13 de março 2021.

[8] Figma Design. Figma: The collaborative interface design tool. Retrieved October, 14,2019

[9] Jakob Nielsen and Rolf Molich. Heuristic evaluation of user interfaces. In Proceedings of the SIGCHI conference on Human factors in computing systems, pages 249-256, 1990

[10] André de Lima Salgado and André Pimenta Freire. Heuristic evaluation of mobile usability: A mapping study. In International Conference on Human-Computer Interaction, pages 178-188. Springer, 2014. 